九州大学学術情報リポジトリ

Kyushu University Institutional Repository

\title{
SECOND-ORDER NECESSARY CONDITIONS FOR AN OPTIMAL CONTROL PROBLEM WITH STATE CONSTRAINTS
}

Maruyama, Yukihiro

Department of Mathematics, Kyushu University

https://doi.org/10.5109/13410

出版情報: Bulletin of informatics and cybernetics. 24 (1/2), pp.53-69, 1990-03. Research Association of Statistical Sciences

バージョン:

権利関係 : 


\title{
SECOND-ORDER NECESSARY CONDITIONS FOR AN OPTIMAL CONTROL PROBLEM WITH STATE CONSTRAINTS
}

By

\author{
Yukihiro MarUYAMA*
}

\begin{abstract}
We study second-order necessary conditions for an optimal control problem with state constraints. The necessary conditions involve an extra term besides the second derivative of the Lagrange function in the sense of mathematical programming. The extra term is expressed as the Lebesgue integral of some upper-semi continuous function.
\end{abstract}

\section{Introduction}

In this paper we are concerned with the following optimal control problem with state constraints which will be referred to as problem (OCP): find the pair of a trajectory function $x \in W_{1,1}^{n}([0, T])$ and a control function $u \in L_{\infty}^{r}([0, T])$ which minimizes the integral functional

$$
\int_{0}^{T} \Phi(t, x(t), u(t)) d t
$$

subject to the constraints

$$
\begin{gathered}
\dot{x}(t)=\varphi(t, x(t), u(t)) \quad \text { a.e. } t \in[0, T], \\
x(0)=x^{0}, \quad x(T)=x^{1}, \\
u(t) \in C \subset \mathbb{R}^{r} \text { a.e. } t \in[0, T], \\
S(t, x(t)) \leqq 0 \text { for all } t \in[0, T] .
\end{gathered}
$$

Here the functions $\Phi: \mathbb{R} \times \mathbb{R}^{n} \times \mathbb{R}^{r} \rightarrow \mathbb{R}, \varphi: \mathbb{R} \times \mathbb{R}^{n} \times \mathbb{R}^{r} \rightarrow \mathbb{R}^{n}$ and $S: \mathbb{R} \times \mathbb{R}^{n} \rightarrow \mathbb{R}$ are assumed to be twice continuously differentiable. The admissible control set $C \subset \mathbb{R}^{r}$ is a closed convex set with int $C \neq \phi$. The end-time $T>0$ is fixed. The symbol $W_{1,1}^{n}([0, T])$ denotes the space of $\mathbb{R}^{n}$-valued, absolutely continuous functions on $[0, T]$ and $L_{\infty}^{r}([0, T])$ denotes the space of $\mathbb{R}^{r}$-valued, measurable, essentially bounded functions on $[0, T]$.

Many authors have adopted the approach of converting the control problem (OCP) into a nonlinear programming problem in Banach spaces. For example, first-order

\footnotetext{
* Department of Mathematics, Kyushu University, Fukuoka, Japan
} 
necessary conditions for a (weak) local minimum solution (Definition 4.1) of the problem (OCP) have been studied by Jacobson, Lele and Speyer [7], Norris [14], Girsanov [5] and Maurer [13]. Second-order necessary conditions have been studied by Warga [15], Girbert and Bernstein [4] and Maruyama [11]. In [11] and [15], the state constraints (1.5) do not appear. In [4], infinitely many inequality constraints (1.5) are replaced by finitely many inequality constraints. In [4], the authors showed that the second derivative of the Lagrange function in the sense of mathematical programming is nonnegative on the set of critical directions. Their results are satisfactory for the problem with finitely many inequality constraints but unsatisfactory for the case of infinitely many inequality constraints because of a phenomenon which is called the envelope-like effect. Kawasaki, in [8], first became aware of this phenomenon for a nonlinear programming problem in Banach spaces and derived new second-order necessary conditions accompanied by their applications to minimizing suptype functions. It should be noted that in [8] set constraints such as (1.4) are not included. In [12], the author obtained new necessary conditions for a nonlinear programming problem which includes a set constraint in addition to infinitely many inequality constraints and equality constraints in a different way from [8].

Our main purpose in the present paper is to investigate the envelope-like effect for the optimal control problem (OCP). We begin in section 2 with reviewing secondorder necessary conditions given in [12]. In section 3, we will give a characterization of a variational set which is involved in the necessary conditions. In section 4, we will apply the necessary conditions stated in section 2 to the optimal control problem (OCP) and derive an important theorem (Theorem 4.1). The second-order necessary condition in Theorem 4.1 involves an extra term besides the second derivative of the Lagrange function. Moreover, we will calculate the extra term in Example 4.1.

\section{Second-Order Necessary Conditions for a Nonlinear Programming Problem in Banach Spaces}

The following nonlinear problem will be referred to as problem $(\mathrm{P})$ : find $x$ which minimizes $f(x)$ subject to the constraints $g(x) \in K, h(x)=0$ and $x \in Q$, where $X, Y$ and $Z$ are Banach spaces, $f: X \rightarrow \mathbb{R}, g: X \rightarrow Y$ and $h: X \rightarrow Z$ are twice Fréchet differentiable, $K$ is a closed convex cone of $Y$ with nonempty interior and $Q$ is a closed convex set of $X$ with nonempty interior.

Kawasaki, in [8], has derived new second-order necessary conditions for the problem (P) without the set constraint $(x \in Q)$. The necessary conditions are of the Kuhn-Tucker type and involve an extra term besides the second derivative of the Lagrange function. The extra term is expressed as a support function of a set $K(u, v)$ (Definition 3.3). The author, in [12], has obtained new second-order necessary conditions for the problem (P) with set constraint $(x \in Q)$. The conditions are of the Fritz-John type and involve an extra term which is expressed as a support function of a set $F(K ; u, v)$ (Definition 2.1).

In this section, we derive a necessary condition of the Kuhn-Tucker type from the condition given in [12] by assuming the Slater-condition (Definition 2.2). 
Let $h: X \rightarrow Z$ be a mapping. Then $D h\left(x_{0}\right)$ and $D^{2} h\left(x_{0}\right)$ denote the first and second Fréchet derivative of $h$ at $x_{0}$, respectively.

Let $Y^{*}$ and $Z^{*}$ be topological dual spaces of $Y$ and $Z$, respectively. For any subset $S$ of $Y$, the support function $\delta^{*}: Y^{*} \rightarrow \mathbb{R} \cup\{+\infty\}$ is defined by

$$
\delta^{*}\left(y^{*} \mid S\right)=\sup \left\{\left\langle y^{*}, y\right\rangle \mid y \in S\right\},
$$

where $y^{*} \in Y^{*}$. The polar cone of $K$ is defined by

$$
K^{p}=\left\{y^{*} \in Y^{*} \mid\left\langle y^{*}, y\right\rangle \leqq 0 \quad \text { for all } y \in K\right\} .
$$

Definition 2.1([3], [6]). For $u \in b d K$ and $v \in Y$, the variational set of $K$ at $u$ (with respect to the point $v$ ) is defined by the following:

$F(K ; u, v)=\left\{x \in X \mid \exists\right.$ n.b.d. $U$ of $x, \exists t_{0}>0$ such that

$$
\left.u+t v+t^{2} U \subset K \quad \text { for all } t \in\left(0, t_{0}\right]\right\} .
$$

The following theorem was proved in [12].

THEOREM 2.1 ([12, corollary 3.2]). Let $x_{0}$ be a local minimum solution of $(P)$ and suppose that $D h\left(x_{0}\right)$ is surjective. Then, corresponding to every $x_{1}$ satisfying the conditions

$$
\begin{gathered}
D f\left(x_{0}\right) x_{1} \leqq 0, \\
D g\left(x_{0}\right) x_{1} \in \operatorname{clcone}\left(K-g\left(x_{0}\right)\right), \\
D h\left(x_{0}\right) x_{1}=0, \\
x_{1} \in \operatorname{cone}\left(Q-x_{0}\right),
\end{gathered}
$$

there exist multipliers $\lambda_{0} \geqq 0, y^{*} \in K^{p}, z^{*} \in Z^{*}$, not all zero, such that

$$
\begin{gathered}
\lambda_{0} D f\left(x_{0}\right) x_{1}=0,\left\langle y^{*}, g\left(x_{0}\right)\right\rangle=0,\left\langle y^{*}, D g\left(x_{0}\right) x_{1}\right\rangle=0, \\
\lambda_{0} D f\left(x_{0}\right) x+\left\langle y^{*}, D g\left(x_{0}\right) x\right\rangle+\left\langle z^{*}, D h\left(x_{0}\right) x\right\rangle \geqq 0
\end{gathered}
$$

for all $x \in \operatorname{clcone}\left(Q-x_{0}\right)$ and

$$
\begin{aligned}
& \hat{\lambda}_{0} D^{2} f\left(x_{0}\right)\left(x_{1}, x_{1}\right)+\left\langle y^{*}, D^{2} g\left(x_{0}\right)\left(x_{1}, x_{1}\right)\right\rangle+\left\langle z^{*}, D^{2} h\left(x_{0}\right)\left(x_{1}, x_{1}\right)\right\rangle \\
& \quad \geqq 2 \delta^{*}\left(y^{*} \mid \operatorname{cl} F\left(K ; g\left(x_{0}\right), D g\left(x_{0}\right) x_{1}\right)\right) .
\end{aligned}
$$

In particular, for every $x_{1}$ satisfying the conditions $D f\left(x_{0}\right) x_{1}<0,(2.2),(2.3)$ and (2.4), the necessary conditions (2.5)-(2.7) hold with $\lambda_{0}=0$.

REMARK 2.1. In general, the value of the extra term $\delta^{*}\left(y^{*} \mid c l F\left(K ; g\left(x_{0}\right), D g\left(x_{0}\right) x_{1}\right)\right)$ is non-positive, see Remark 3.3 in [12]. However, if we restrict Theorem 2.1 to the direction $x_{1}$ satisfying $D g\left(x_{0}\right) x_{1} \in \operatorname{cone}\left(K-g\left(x_{0}\right)\right),(2.1),(2.3)$ and (2.4), then the value of the extra term is zero, see Remark 3.5 in [12]. When $Y=\mathbb{R}^{m}$ and $K=\mathbb{B}_{+}^{m}$, the set cone $\left(K-g\left(x_{0}\right)\right)$ is closed. Hence in this case, from Theorem 2.1 and the above explanation, we obtain a well-known second-order necessary condition: the second derivative of the Lagrange function is nonnegative.

REMARK 2.2. Theorem 2.1 requires the assumption that the mappings $f, g$ and $h$ are twice Fréchet differentiable. This assumption can be weakened. The author, in 
[12], has derived a second-order necessary condition by using second-order Neustadt derivative defined by the following; see theorem 3.2 in [12]: Let $\hat{X}, \hat{Y}$ be real Banach spaces. Let $f: \hat{X} \rightarrow \hat{Y}$, and let $x_{0} \in \hat{X}$. If for every point $x_{1}$ of $\hat{X}$ there exists a point $f^{(1)}\left(x_{0} ; x_{1}\right)$ of $\hat{Y}$ such that

$$
f^{(1)}\left(x_{0} ; x_{1}\right)=\lim _{\substack{y \rightarrow x_{1} \\ \lambda \downarrow 0}}(1 / \lambda)\left[f\left(x_{0}+\lambda y\right)-f\left(x_{0}\right)\right]
$$

then we call the mapping $f^{(1)}\left(x_{0} ; \cdot\right)$ the first-order Neustadt derivative of $f$ at $x_{0}$. Let $x_{1}$ be a point of $\hat{X}$. If $f$ has the first-order Neustadt derivative $f^{(1)}\left(x_{0} ; \cdot\right)$, and if for every point $x_{2}$ of $\hat{X}$ there exists a point $f^{(2)}\left(x_{0}, x_{1} ; x_{2}\right)$ of $\hat{Y}$ such that

$$
f^{(2)}\left(x_{0}, x_{1} ; x_{2}\right)=\lim _{\substack{y \rightarrow x_{2} \\ \lambda \downarrow 0}}\left(1 / \lambda^{2}\right)\left[f\left(x_{0}+\lambda x_{1}+\lambda^{2} y\right)-f\left(x_{0}\right)-\lambda f^{(1)}\left(x_{0} ; x_{1}\right)\right],
$$

then we call the mapping $f^{(2)}\left(x_{0}, x_{1} ; \cdot\right)$ the second-order Neustadt derivative of $f$ at $x_{0}$ (with respect to $x_{1}$ ).

The necessary conditions in Theorem 2.1 are of the Fritz-John type. We shall derive second-order necessary conditions of the Kuhn-Tucker type under the following constraint qualification

Definition 2.2. (Maurer[13]). The system

$$
g(x) \in K, \quad h(x)=0, \quad x \in Q
$$

is said to satisfy the Slater condition at $x_{0}$ if

(i) $\operatorname{Dh}\left(x_{0}\right)$ is surjective,

(ii) there exists $\bar{x} \in$ int $Q-x_{0}$ with

$$
\operatorname{Dh}\left(x_{0}\right) \bar{x}=0
$$

and

$$
g\left(x_{0}\right)+D g\left(x_{0}\right) \bar{x} \in \text { int } K .
$$

This condition is found in e.g. [8], [13].

COROLlARY 2.1. In addition to the assumption of Theorem 2.1, we suppose that the system (2.8) satisfies the Slater condition at $x_{0}$ which is a local minimum solution of $(P)$. Then, corresponding to every $x_{1}$ satisfying the conditions

$$
D f\left(x_{0}\right) x_{1}=0,
$$

(2.2), (2.3) and (2.4), there exist multipliers $y^{*} \in K^{p}, z^{*} \in Z^{*}$, not both zero, such that the necessary conditions (2.5)-(2.7) hold with $\lambda_{0}=1$.

PROOF. It follows from (2.6) that

$$
\lambda_{0} D f\left(x_{0}\right) x+\left\langle y^{*}, D g\left(x_{0}\right) x\right\rangle+\left\langle z^{*}, D h\left(x_{0}\right) x\right\rangle \geqq 0
$$

for all $x \in Q-x_{0}$, where $\lambda_{0} \geqq 0$. Suppose that $\lambda_{0}=0$. Then it holds that

$$
\left\langle y^{*}, D g\left(x_{0}\right) x\right\rangle+\left\langle z^{*}, D h\left(x_{0}\right) x\right\rangle \geqq 0
$$

for all $x \in Q-x_{0}$. It follows from (2.10) in the Slater condition that there exist a point 
$\bar{x} \in$ int $Q-x_{0}$ and a neighbourhood $B(0) \subset Y$ of the origin such that

$$
g\left(x_{0}\right)+D g\left(x_{0}\right) \bar{x}+B(0) \subset K .
$$

Hence for all $d \in B(0)$

$$
\left\langle y^{*}, g\left(x_{0}\right)+D g\left(x_{0}\right) \bar{x} \pm d\right\rangle \leqq 0 .
$$

From (2.5) and (2.13), we have

$$
\left\langle y^{*}, D g\left(x_{0}\right) \bar{x}\right\rangle \leqq\left\langle y^{*}, \pm d\right\rangle .
$$

It follows from (2.9) in the Slater condition and (2.12) that

$$
\left\langle y^{*}, D g\left(x_{0}\right) \bar{x}\right\rangle \geqq 0 .
$$

From (2.14) and (2.15), it holds that $y^{*}=0$. Hence it follows from (2.12) that

$$
\left\langle z^{*}, D h\left(x_{0}\right) x\right\rangle \geqq 0
$$

for all $x \in Q-x_{0}$. Moreover, since $\bar{x} \in$ int $Q-x_{0}$, there exists a neighbourhood $\hat{B}(0)$ such that $\bar{x}+\hat{B}(0) \subset Q-x_{0}$. Hence from (2.15), we have that

$$
\left\langle z^{*}, D h\left(x_{0}\right)(\bar{x} \pm \hat{d})\right\rangle \geqq 0 \quad \text { for all } \hat{d} \in \hat{B}(0) .
$$

Since $\operatorname{Dh}\left(x_{0}\right) \bar{x}=0$, it follows from (2.17) that for all $\hat{d} \in \hat{B}(0)$

$$
\left\langle z^{*}, D h\left(x_{0}\right) \hat{d}\right\rangle=0 \text {. }
$$

Since $\operatorname{Dh}\left(x_{0}\right)$ is an onto mapping, $z^{*}=0$. Consequently, we have that $\left(\lambda_{0}, y^{*}, z^{*}\right)=$ $(0,0,0)$, which is contradiction. Hence we can take $\lambda_{0}=1$.

REMARK 2.3. One may think that Theorem 2.1 is stronger than Corollary 2.1 because the latter states nothing about the directions satisfying $D f\left(x_{0}\right) x_{1}<0,(2.2),(2.3)$ and (2.4). But it is similarly proved as the above proof that such a direction does not exist under the Slater condition. Hence Corollary 2.1 is not weaker than Theorem 2.1 with the added restriction of the Slater condition.

\section{Characterization of Variational Sets}

Let $I$ be a compact set in a metric space. Then the set of all real valued continuous functions on $I$ is denoted by $C(I)$.

In this section, we study the relation between some variational sets and characterize the variational set $\operatorname{cl} F(K ; u, v)$ when $K=C_{+}(I)$, where

$$
C_{+}(I)=\{y \in C(I) \mid y(t) \geqq 0 \text { for all } t \in I\} .
$$

Definition 3.1. For any $u, v \in C(I)$, the set $\Gamma(u, v)$ is defined by

$$
\Gamma(u, v)=\left\{x \in C(I) \mid \exists s_{0}>0 \text { s.t. } u+s v+s^{2} x \in C_{+}(I) \text { for all } s \in\left(0, s_{0}\right]\right\} .
$$

The set $\Gamma(u, v)$ was examined in [1] and [2]. The set $c l \Gamma(u, v)$ is called the set of admissible second-order directions. We derive the relation between the set $\Gamma(u, v)$ and the variational set $F\left(C_{+}(I) ; u, v\right)$. 
LEMMA 3.1. For any $u, v \in C(I)$, the following relation holds.

$$
c l F\left(C_{+}(I) ; u, v\right)=c l \Gamma(u, v) .
$$

Proof. Clearly $c l F\left(C_{+}(I) ; u, v\right) \subset c l \Gamma(u, v)$. Hence in order to obtain (3.2), it suffices to show that

$$
\Gamma(u, v) \subset \operatorname{clF}\left(C_{+}(I) ; u, v\right) .
$$

Let $x \in \Gamma(u, v)$. Then there exists $s_{0}>0$ such that

$$
u+s v+s^{2} x \in C_{+} \quad \text { for all } s \in\left(0, s_{0}\right] .
$$

Hence there exists $\{k(s)\}_{s \in\left(0, s_{0}\right]} \subset C_{+}(I)$ such that

$$
x=k(s)-u / s^{2}-v / s \quad \text { for all } s \in\left(0, s_{0}\right] .
$$

Let $\left\{k_{n}\right\}$ be a sequence of int $C_{+}(I)$ such that $k_{n} \downarrow 0$. Then for each $k_{n}$, there exists a neighbourhood $U_{n}(0)$ such that $k_{n}+U_{n}(0) \subset$ int $C_{+}(I)$. Hence we have that

$$
x+k_{n}+d=k(s)-u / s^{2}-v / s+k_{n}+d \in C_{+}(I)-u / s^{2}-v / s,
$$

for all $d \in U_{n}(0)$. Put $x+k_{n}=y_{n}$. Then $y_{n} \in F\left(C_{+}(I) ; u, v\right)$ for each $n$ and $y_{n} \rightarrow x$ as $n \rightarrow+\infty$. Consequently the relation (3.3) follows.

The following lemma was proved by Kawasaki.

LEMma $3.2([8])$. Let $u \in C_{+}(I)$. Then $v \in \operatorname{cl}\left(\operatorname{cone}\left(C_{+}(I)-u\right)\right)$ if and only if

$$
v(t) \geqq 0 \quad \text { for all } t \text { satisfying } u(t)=0 .
$$

The functional $E(t)$ and the set $K(u, v)$ were also defined by Kawasaki.

Definition 3.2 ([9]). Let $T_{0}$ denote the set of all $t \in I$ for which there exists a sequence $\left\{t_{n}\right\} \subset I$ satisfying (3.5) below

$$
u\left(t_{n}\right)>0, t_{n} \rightarrow t \text { and }-v\left(t_{n}\right) / u\left(t_{n}\right) \rightarrow+\infty \text { as } n \rightarrow+\infty .
$$

Then the functional $E(t)$ is defined by

$$
E(t)=\left\{\begin{aligned}
\sup \left\{\limsup v\left(t_{n}\right)^{2} / 4 u\left(t_{n}\right) \mid\left\{t_{n}\right\} \text { satisfies }(3.5)\right\}, & \text { if } t \in T_{0}, \\
0, & \text { if } u(t)=v(t)=0 \text { and } t \in T_{0}, \\
-\infty, & \text { otherwise }
\end{aligned}\right.
$$

Definition 3.3 ([8]). For any $u, v \in C(I)$, the set $K(u, v)$ is defined by

$$
K(u, v)=\left\{x \in C(I) \mid \exists \Delta(\theta) \text { s.t. } \theta^{2} u+\theta v+x+\Delta(\theta) \in C_{+}(I) \text { for all } \theta>0\right\} .
$$

where $\Delta(\theta)$ is an arbitrary element of $C(I)$ satisfying $\Delta(\theta) \rightarrow 0$ as $\theta \rightarrow+\infty$.

Kawasaki has given the characterization of $K(u, v)$ as follows: Let $u \in C_{+}(I)$ and $v$ satisfy (3.4). Then it holds that

$$
K(u, v)=\{x \in C(I) \mid x(t) \geqq E(t) \text { for all } t \in I\},
$$

see theorem 3.2 in [9]. 
Definition 3.4. For any $u, v \in C(I)$, the set $K^{0}(u, v)$ is defined by

$$
K^{0}(u, v)=\{x \in C(I) \mid x(t)>E(t) \text { for all } t \in I\} .
$$

The relation between $K^{0}(u, v)$ and $\Gamma(u, v)$ is as follows.

LemMa 3.3. Let $u \in C_{+}(I)$ and $v$ satisfy (3.4). Then it holds that

$$
K^{0}(u, v) \subset \Gamma(u, v) \text {. }
$$

Proof. Deny (3.10), then there exists $x \in C(I)$ satisfying that

$$
\begin{aligned}
& x \in K^{0}(u, v), \\
& x \notin \Gamma(u, v) .
\end{aligned}
$$

By (3.12), there exists $\left\{s_{n}\right\} \downarrow 0$ such that $u+s_{n} v+s_{n}^{2} x \notin C_{+}(I)$. Hence, for each $n$, there exists $t_{n} \in I$ such that

$$
u\left(t_{n}\right)+s_{n} v\left(t_{n}\right)+s_{n}^{2} x\left(t_{n}\right)<0 .
$$

Since $I$ is compact, there exists a subsequence $\left\{t_{n}\right\}$ satisfying that $t_{n} \rightarrow t \in I$. Put $1 / s_{n}=\theta_{n}$. Then it follows from (3.13) that

$$
\theta_{n}^{2} u\left(t_{n}\right)+\theta_{n} v\left(t_{n}\right)+x\left(t_{n}\right)<0
$$

for all $n$ and $\theta_{n} \rightarrow+\infty$. Suppose that infinitely many $t_{n}$ would satisfy $u\left(t_{n}\right)=0$. Then, from (3.4) and (3.14), we have $x(t) \leqq E(t)=0$, this contradicts (3.11). So we may assume that $u\left(t_{n}\right)>0$ for sufficiently large $n$. Then from (3.14), the discriminant $D_{n}=$ $v\left(t_{n}\right)^{2}-4 u\left(t_{n}\right) x\left(t_{n}\right)$ is positive, that is,

$$
x\left(t_{n}\right)<v\left(t_{n}\right)^{2} / 4 u\left(t_{n}\right) \quad \text { for all } n .
$$

Taking $n \rightarrow+\infty$, we get

$$
x(t) \leqq \limsup _{n \rightarrow+\infty} v\left(t_{n}\right)^{2} / 4 u\left(t_{n}\right) .
$$

If we show that $-v\left(t_{n}\right) / u\left(t_{n}\right) \rightarrow+\infty$ as $n \rightarrow+\infty$, then $x(t) \leqq E(t)$ contradicting (3.11), thus, the proof of (3.10) is completed. Solving (3.14), we have that

$$
\theta_{n}<\left(-v\left(t_{n}\right)+\sqrt{D_{n}}\right) / 2 u\left(t_{n}\right) .
$$

Since $\theta_{n} \rightarrow+\infty$, we have $u\left(t_{n}\right) \rightarrow 0$. Hence $u(t)=0$, so that $v(t) \geqq 0$. It is clear that

$$
\left(-v\left(t_{n}\right)+\sqrt{D_{n}}\right) / 2 u\left(t_{n}\right)=\left(-2 x\left(t_{n}\right)\right) /\left(v\left(t_{n}\right)+\sqrt{D_{n}}\right) .
$$

The denominator of the right side of (3.16) converges to $v(t)+|v(t)|$, which is equal to $2 v(t)$ because $v(t) \geqq 0$. Since the left side of (3.16) tends to $+\infty$, it holds that $v(t)=0$. So, from (3.11), we have $x(t)>0$. Thus, there exists $n_{0}$ such that

$$
x\left(t_{n}\right) \geqq 0
$$

for all $n \geqq n_{0}$. It follows from (3.15) and (3.17) that

$$
\theta_{n}<\left(-v\left(t_{n}\right)+\sqrt{D_{n}}\right) / 2 u\left(t_{n}\right) \leqq\left(-v\left(t_{n}\right)+\left|v\left(t_{n}\right)\right|\right) / 2 u\left(t_{n}\right)
$$


for all $n \geqq n_{0}$. Since $\theta_{n}$ tends to $+\infty$, we have that $v\left(t_{n}\right)<0$ and $\left(-v\left(t_{n}\right)\right) / u\left(t_{n}\right) \rightarrow+\infty$ as $n \rightarrow+\infty$. This completes the proof of lemma.

We now give a characterization of the variational set $c l F\left(C_{+}(I) ; u, v\right)$.

THEOREM 3.1. Let $u, v$ satisfy the assumption of Lemma 3.3. Then

$$
c l F\left(C_{+}(I) ; u, v\right)=\{x \in C(I) \mid x(t) \geqq E(t) \text { for all } t \in I\} .
$$

Proof. We first show that

$$
c l \Gamma(u, v) \subset K(u, v) .
$$

Since $K(u, v)$ is closed, it suffices to show that

$$
\Gamma(u, v) \subset K(u, v) .
$$

Let $x \in \Gamma(u, v)$. Then there exists $s_{0}>0$ such that

$$
u+s v+s^{2} x \subset C_{+}(I) \quad \text { for all } s \in\left(0, s_{0}\right] .
$$

Since $C_{+}(I)$ is cone, it holds that

$$
\left(1 / s^{2}\right) u+(1 / s) v+x \in C_{+}(I) \quad \text { for all } s \in\left(0, s_{0}\right] .
$$

Put $\theta=1 / s$ and define $\Delta(\theta)$ as follows:

$$
\Delta(\theta)= \begin{cases}0 & \text { if } \theta \geqq 1 / s_{0}, \\ -x-\theta v-\theta^{2} u & \text { if } \theta<1 / s_{0},\end{cases}
$$

Then it follows from (3.20) that

$$
\theta^{2} u+\theta v+x+\Delta(\theta) \in C_{+}(I) \quad \text { for all } \theta>0
$$

and $\Delta(\theta) \rightarrow 0$ as $\theta \rightarrow+\infty$. So, by definition, $x \in K(u, v)$, proving (3.19). Moreover it is easy to show that

$$
\text { int } K(u, v) \subset K^{0}(u, v) \text {. }
$$

It follows from (3.10) in Lemma 3.3, (3.19) and (3.21) that

$$
K(u, v) \subset c l K^{0}(u, v) \subset c l \Gamma(u, v) \subset K(u, v) .
$$

So

$$
K(u, v)=c l \Gamma(u, v)=c l K^{0}(u, v) .
$$

Hence it follows from (3.2) in Lemma 3.1 that

$$
c l F\left(C_{+}(I) ; u, v\right)=K(u, v) .
$$

So (3.18) follows from (3.8).

\section{Second-Order Necessary Conditions for the Optimal Control Problem with State Constraints}

In this section we apply the necessary conditions stated in section 2 to the optimal control problem (OCP). We derive new necessary conditions for the problem (OCP) 
with state constraints by using the characterization theorem of the variational set $c l F\left(C_{+}([0, T]) ; u, v\right)$. We start with converting the control problem (OCP) into the nonlinear problem $(\mathrm{P})$.

Let

$$
\begin{aligned}
& X=W_{1,1}^{n}([0, T]) \times L_{\infty}^{r}([0, T]), \quad Y=C([0, T]), \\
& K=C_{+}([0, T]), \quad Z=L_{1}^{n}([0, T]) \times \mathbb{R}^{n} \times \mathbb{R}^{n} .
\end{aligned}
$$

The space $X$ is endowed with the following norm

$$
\|(x, u)\|=\max \left\{\|x\|_{1},\|\dot{x}\|_{1},\|u\|_{\infty}\right\} .
$$

Here $\|x\|_{1}$ and $\|\dot{x}\|_{1}$ are $L_{1}$-norms defined in the usual way using the euclidean norm in $\mathbb{R}^{n}$ and $\|u\|_{\infty}$ is $L_{\infty}$-norm defined using the euclidean norm in $\mathbb{R}^{r}$. The space $X$ endowed with the norm (4.2) is a Banach space. Since $C$ is closed and convex with int $C \neq \phi$, the set

$$
Q=\{(x, u) \in X \mid u(t) \in C \text { a.e. } t \in[0, T]\}
$$

is closed and convex with int $Q \neq \phi$ (See [13]). Define the functions $f: X \rightarrow \mathbb{R}$, $g: X \rightarrow Y$ and $h: X \rightarrow Z$ by

$$
\begin{gathered}
f(x, u)=\int_{0}^{T} \Phi(t, x(t), u(t)) d t, \\
{[g(x, u)](t)=-S(t, x(t)),} \\
{[h(x, u)](t)=\left(\dot{x}(t)-\varphi(t, x(t), u(t)), x(0)-x^{0}, x(T)-x^{1}\right) .}
\end{gathered}
$$

By means of (4.1), (4.3)-(4.6) the control problem (OCP) can be cast into the nonlinear programming problem $(\mathrm{P})$ with which we have been concerned in section 2 .

In what follows all vectors are column vectors. The transpose of vectors or matrices is denoted by an asterisk. The Jacobian matrices of partial derivatives with respect to $x$ and $u$ are denoted by subscripts. Let $\left(x_{0}, u_{0}\right)$ be a point of the feasible set $M$ determined by the constraints (1.2)-(1.5). For simplicity arguments of functions involving $x_{0}(t), u_{0}(t)$ will be abbreviated by $[t]$; for instance $\Phi[t]=\Phi\left(t, x_{0}(t), u_{0}(t)\right)$.

In the following, we are concerned with a weak local minimum solution.

Definition 4.1. Let $\left(x_{0}, u_{0}\right) \in M$. We shall say that $\left(x_{0}, u_{0}\right)$ is a local minimum solution of the optimal control problem (OCP) if there exists a number $\varepsilon>0$ such that the inequality $f\left(x_{0}, u_{0}\right) \leqq f(x, u)$ holds for all $(x, u) \in M$ satisfying $\left\|(x, u)-\left(x_{0}, u_{0}\right)\right\|<\varepsilon_{0}$.

For brevity, we denote the pair $(x, u)$ in $X$ by $z$ and denote the space $C_{+}([0, T])$ by $C_{+}$.

We now review the polar cone of $C_{+}$. Any element $y^{*}$ of $\left(C_{+}\right)^{p}$ is represented as

$$
\left\langle y^{*}, y\right\rangle=-\int_{0}^{T} y(t) d \psi(t),
$$

where $\psi$ is a certain nondecreasing function. Let $\Psi$ denote the measure induced from $\psi$. For the problem (OCP), the complementary condition (2.10) becomes as follows; see Lemma 4.2 in [9]: 
Lemma 4.1. Let $g\left(z_{0}\right) \in C_{+}, D g\left(z_{0}\right) z_{1} \in \operatorname{clcone}\left(C_{+}-g\left(z_{0}\right)\right)$. Then it holds that

(i) $\left\langle y^{*}, g\left(z_{0}\right)\right\rangle=0$ if and only if $\Psi\left(\left\{t \mid\left[g\left(z_{0}\right)\right](t)>0\right\}\right)=0$,

(ii) $\left\langle y^{*}, D g\left(z_{0}\right) z_{1}\right\rangle=0 \quad$ if and only if $\Psi\left([0, T] \backslash\left\{t \mid\left[g\left(z_{0}\right)\right](t)=\left[D g\left(z_{0}\right) z_{1}\right](t)=0\right\}\right)=0$

We denote the functional $E(t)$ and $T_{0}$ defined for $u=g\left(z_{0}\right)$ and $v=D g\left(z_{0}\right) z_{1}$ by $E\left(t ; z_{0}, z_{1}\right)$ and $T_{0}\left(z_{0}, z_{1}\right)$, respectively.

Combining lemma 4.4 in [9] and Theorem 3.1, we obtain the following lemma.

LEMMA 4.2. Let $g\left(z_{0}\right)$ and $D g\left(z_{0}\right) z_{1}$ satisfy the assumption of Lemma 4.1. Then it holds that for $y^{*} \in\left(C_{+}\right)^{p}$

$$
\delta^{*}\left(y^{*} \mid \operatorname{cl} F\left(C_{+} ; g\left(z_{0}\right), D g\left(z_{0}\right) z_{1}\right)\right)=-\int_{0}^{T} E\left(t ; z_{0}, z_{1}\right) d \Psi^{*}(t),
$$

where $\Psi^{*}$ is the completion of the measure $\Psi$.

Proof. It follows from (3.18) in Theorem 3.1 that

$$
\operatorname{clF}\left(C_{+} ; g\left(z_{0}\right), D g\left(z_{0}\right) z_{1}\right)=\left\{y \in C([0, T]) \mid y(t) \geqq E\left(t ; z_{0}, z_{1}\right) \text { for all } t \in[0, T]\right\} .
$$

From (4.7) and (4.11), it holds that

$$
\begin{aligned}
\delta^{*} & \left(y^{*} \mid \operatorname{clF}\left(C_{+} ; g\left(z_{0}\right), D g\left(z_{0}\right) z_{1}\right)\right) \\
& =\sup \left\{\left\langle y^{*}, y\right\rangle \mid y \in \operatorname{clF}\left(C_{+} ; g\left(z_{0}\right), D g\left(z_{0}\right) z_{1}\right)\right\} \\
& =-\inf \left\{\int_{0}^{T} y(t) d \psi(t) \mid y(t) \geqq E\left(t ; z_{0}, z_{1}\right) \text { for all } t \in[0, T]\right\} . \\
& =-\int_{0}^{T} E\left(t ; z_{0}, z_{1}\right) d \Psi^{*}(t) .
\end{aligned}
$$

Consequently, the relation (4.10) follows.

We are now in a position to state our second-order necessary condition for the optimal control problem (OCP).

THEOREM 4.1. Let $z_{0}=\left(x_{0}, u_{0}\right)$ be a local minimum solution of the optimal control problem $(O C P) . \quad$ Moreover we assume that the linearized system $\dot{x}=\varphi_{x}[t] x+\varphi_{u}[t] u$ is completely controllable (see [5]).

Then, corresponding to every $z_{1}=\left(x_{1}, u_{1}\right)$ satisfying the conditions

$$
\begin{gathered}
\int_{0}^{T}\left\{\Phi_{x}[t] x_{1}(t)+\Phi_{u}[t] u_{1}(t)\right\} d t \leqq 0, \\
\max _{t \in I\left(x_{0}\right)} S_{x}\left(t, x_{0}(t)\right) x_{1}(t) \leqq 0, \\
\dot{x}_{1}(t)=\varphi_{x}[t] x_{1}(t)+\varphi_{u}[t] u_{1}(t), \\
x_{1}(0)=x_{1}(T)=0, \\
u_{1} \in\left\{\hat{\lambda}\left(u-u_{0}\right) \mid \hat{\lambda}>0, u \in L_{\infty}^{r}([0, T]), u(t) \in C \text { a.e. } t \in[0, T]\right\},
\end{gathered}
$$

there exist $\lambda_{0} \geqq 0$, a nondecreasing function $\psi \in B V[0, T]$ and $\lambda \in L_{\infty}^{n}([0, T])$, not all 
zero, such that

$$
\Psi\left([0, T] \backslash I\left(z_{0}\right)\right)=0, \quad \Psi\left([0, T] \backslash I\left(z_{0}, z_{1}\right)\right)=0,
$$

[local minimal principle]

(i) $-\lambda(t)^{*}=-c+\int_{t}^{T} H_{x}[s] d s+\int_{t}^{T} S_{x}[s] d \psi(s)$

(ii) $H_{u}[t]\left(u-u_{0}(t)\right) \geqq 0$

for all $u \in C$ and a.e. $t \in[0, T]$,

[second-order necessary condition]

$$
\begin{aligned}
& \int_{0}^{T}\left(x_{1}(t), u_{1}(t)\right)^{*}\left(\begin{array}{l}
H_{x x}[t], H_{x u}[t] \\
H_{u x}[t], H_{u u}[t]
\end{array}\right)\left(\begin{array}{l}
x_{1}(t) \\
u_{1}(t)
\end{array}\right) d t \\
& \quad+\int_{0}^{T} x_{1}(t)^{*} S_{x x}[t] x_{1}(t) d \psi(t)+2 \int_{0}^{T} E\left(t ; z_{0}, z_{1}\right) d \Psi^{*}(t) \geqq 0,
\end{aligned}
$$

where

$$
\begin{aligned}
I\left(z_{0}\right) & =\left\{t \in[0, T] \mid S\left(t, x_{0}(t)\right)=0\right\} \\
I\left(z_{0}, z_{1}\right) & =\left\{t \in[0, T] \mid S\left(t, x_{0}(t)\right)=S_{x}\left(t, x_{0}(t)\right) x_{1}(t)=0\right\}
\end{aligned}
$$

and $H$ is the Hamiltonian function, that is,

$$
H\left(t, x, y, \lambda_{0}, \lambda\right)=\lambda_{0} \Phi(t, x, y)-\lambda(t)^{*} \varphi(t, x, y) .
$$

Proof. The mappings $f, g$ and $h$ defined by (4.4)-(4.6) are twice Fréchet differentiable at $z_{0}\left(z_{0}=\left(x_{0}, u_{0}\right)\right)$. First and second-order Fréchet derivatives of $f$ are as follows:

$$
\begin{gathered}
D f\left(z_{0}\right) z=\int_{0}^{T}\left\{\Phi_{x}[t] x(t)+\Phi_{u}[t] u(t)\right\} d t \\
D f^{2}\left(z_{0}\right)\left(z_{1}, z_{1}\right)=\int_{0}^{T}\left(x_{1}(t), u_{1}(t)\right)^{*}\left(\begin{array}{l}
\Phi_{x x}[t], \Phi_{x u}[t] \\
\Phi_{u x}[t], \Phi_{u u}[t]
\end{array}\right)\left(\begin{array}{l}
x_{1}(t) \\
u_{1}(t)
\end{array}\right) d t
\end{gathered}
$$

First and second-order Fréchet derivatives of $g$ are as follows:

$$
\begin{aligned}
{\left[D g\left(z_{0}\right) z\right](t) } & =-S_{x}\left(t, x_{0}(t)\right) x(t), \\
{\left[D^{2} g\left(z_{0}\right)\left(z_{1}, z_{1}\right)\right](t) } & =-x_{1}(t)^{*} S_{x x}\left(t, x_{0}(t)\right) x_{1}(t) .
\end{aligned}
$$

First and second-order Fréchet derivatives of $h$ are as follows:

$$
\begin{aligned}
{\left[D h\left(z_{0}\right) z\right](t) } & =\left(\dot{x}(t)-\left(\varphi_{x}[t] x(t)+\varphi_{u}[t] u(t)\right), x(0), x(T)\right), \\
{\left[D^{2} h\left(z_{0}\right)\left(z_{1}, z_{1}\right)\right](t) } & =-\left(x_{1}(t), u_{1}(t)\right) *\left(\begin{array}{l}
\varphi_{x x}[t], \varphi_{x u}[t] \\
\varphi_{u x}[t], \varphi_{u u}[t]
\end{array}\right)\left(\begin{array}{l}
x_{1}(t) \\
u_{1}(t)
\end{array}\right) .
\end{aligned}
$$

By Theorem 2.1 we have that corresponding to every $z_{1}$ satisfying the conditions

$$
D f\left(z_{0}\right) z_{1} \leqq 0,
$$




$$
\begin{gathered}
\operatorname{Dg}\left(z_{0}\right) z_{1} \in \operatorname{clcone}\left(C_{+}-g\left(z_{0}\right)\right), \\
\operatorname{Dh}\left(z_{0}\right) z_{1}=0 \\
z_{1} \in \operatorname{cone}\left(Q-z_{0}\right),
\end{gathered}
$$

there exist multipliers $\lambda_{0} \geqq 0, y^{*} \in\left(C_{+}\right)^{p}, z^{*} \in Z^{*}$, not all zero, such that

$$
\begin{gathered}
\left\langle y^{*}, g\left(z_{0}\right)\right\rangle=0, \quad\left\langle y^{*}, D g\left(z_{0}\right) z_{1}\right\rangle=0, \\
\lambda_{0} D f\left(z_{0}\right) z+\left\langle y^{*}, D g\left(z_{0}\right) z\right\rangle+\left\langle z^{*}, D h\left(z_{0}\right) z>\geqq 0\right.
\end{gathered}
$$

for all $z \in \operatorname{cone}\left(Q-z_{0}\right)$ and

$$
\begin{aligned}
& \lambda_{0} D^{2} f\left(z_{0}\right)\left(z_{1}, z_{1}\right)+\left\langle y^{*}, D^{2} g\left(z_{0}\right)\left(z_{1}, z_{1}\right)\right\rangle+\left\langle z^{*}, D^{2} h\left(z_{0}\right)\left(z_{1}, z_{1}\right)\right\rangle \\
& \quad \geqq 2 \delta^{*}\left(y^{*} \mid c l F\left(C_{+} ; g\left(z_{0}\right), D g\left(z_{0}\right) z_{1}\right)\right) .
\end{aligned}
$$

It is clear from (4.20) and (4.24) that (4.26) and (4.28) are equivalent to (4.12) and (4.14), respectively. Moreover, by Lemma 3.2, the condition (4.27) holds if and only if

$$
-S_{x}\left(t, x_{0}(t)\right) x_{1}(t) \geqq 0 \quad \text { for all } t \in I\left(x_{0}\right),
$$

which is equivalent to (4.13).

The complementary condition (4.30) is reduced to (4.16), see Lemma 4.1.

According to the definition of the space $Z$, a Lagrange multiplier $y^{*} \in Z^{*}$ splits into

$$
y^{*}=\left(\lambda^{*}, c_{1}, c_{2}\right) \in\left(L_{1}^{n}([0, T])\right)^{*} \times \mathbb{R}^{n} \times \mathbb{R}^{n} .
$$

Moreover the linear functional $\lambda^{*}$ has the following representation:

$$
\left\langle\lambda^{*}, z\right\rangle=\int_{0}^{T} \lambda(t)^{*} z(t) d t \quad \text { for } z \in L_{1}^{n}([0, T]),
$$

where $\lambda \in L_{\infty}^{n}([0, T])$. Hence it follows from (4.20), (4.22), (4.24) (4.33) and (4.34) that the first-order necessary condition $(4.31)$ is equivalent to

$$
\begin{aligned}
& \lambda_{0} \int_{0}^{T}\left\{\Phi_{x}[t] x(t)+\Phi_{u}(t) u(t)\right\} d t+\int_{0}^{T} S_{x}[t] x(t) d \psi(t) \\
& \quad+\int_{0}^{T} \lambda(t)^{*}\left\{\dot{x}(t)-\left(\varphi_{x}[t] x(t)+\varphi_{u}[t] u(t)\right)\right\} d t+c_{1}^{*} x(0)+c_{2}^{*} x(T) \geqq 0
\end{aligned}
$$

for all $x \in W_{1,1}^{n}([0, T])$ and all $u \in\left\{\hat{\lambda}\left(u-u_{0}\right) \mid \lambda>0, u(t) \in C\right.$ a.e. $\left.t\right\}$. From this the local minimum principle (4.17) and (4.18) are derived, see [11].

Finally, by (4.10) in lemma 4.2, (4.21), (4.23), (4.25) and (4.32), we obtain the second-order necessary condition (4.19). This completes the proof of the theorem.

First-order necessary conditions (4.17) and (4.18) are called the local minimum principle in [5] and [13]. Second-order necessary conditions involving the extra term appeared in the other literature [8], [9] and [12], but are new in the theory of optimal control. In general, the value of the extra term $2 \int_{0}^{T} E\left(t ; z_{0}, z_{1}\right) d \Psi^{*}(t)$ is non-negative, 
see Remark 2.1. This term is considered as the result of the envelope-like effect in the optimal control problem (OCP) with state constraints. The envelope-like effect does not appear if, in the problem (OCP), the state constraints are replaced by finitely many inequality constraints. We shall pursue this issue in detail in the following remark.

REMARK 4.1. Let the state constraints in (OCP) be replaced by the following:

$$
S_{i}(x(0), x(T)) \leqq 0, \quad i \in\{1, \ldots, m\},
$$

where $S_{i}: \mathbb{R}^{n} \times \mathbb{R}^{n} \rightarrow \mathbb{R}$, for $i \in\{1, \ldots, m\}$, and delete the condition (1.3). Put $g(x)=$ $\left(-S_{1}(x(0), x(T)), \ldots,-S_{m}(x(0), x(T))\right)$. Then inequality constraints (4.36) are reduced to the relation such that $g(x) \in \mathbb{R}_{+}^{m}$. Hence, from Theorem 4.1 and the same discussion as Remark 2.1, we obtain a second-order necessary condition such that the second derivative of the Lagrange function is nonnegative; this necessary condition involves no extra term. Consequently, the envelope-like effect disappear in the control problem with finitely many inequality constraints (4.36).

The Slater condition for the problem (OCP) is the following.

Condition 4.1 (Maurer [13]).

(i) The linearized system $\dot{x}=\varphi_{x}[t] x+\varphi_{u}[t] u$ is completely controllable,

(ii) there exists a control $u$ and $\varepsilon>0$ such that

(a) $B_{\varepsilon}(u(t)) \subset C-u_{0}(t)$ a.e. $t \in[0, T]$,

(b) $S[t]+S_{x}[t] x(t)<0$ for all $t \in[0, T]$, where $x$ satisfies $\dot{x}=\phi_{x}[t] x+$ $\phi_{u}[t] u, x(0)=x(T)=0$.

This condition is found in [13]. From Corollary 2.1, we obtain the following.

COROLlaRY 4.1. In addition to the assumptions of Theorem 4.1, suppose that the Condition 4.1 is satisfied. Then the multiplier $\lambda_{0}$ is not zero.

REMARK 4.3. For an optimal control problem with a free right end point and a fixed time, the necessary condition (4.17) holds with $c=0$, that is

$$
-\lambda(t)^{*}=\int_{t}^{T} H_{x}[s] d s+\int_{t}^{T} S_{x}[s] d \psi(s) .
$$

We now compute the extra term in the following problem which is regarded as a production-planning problem with no inventory costs but with known demand, see example 4 in $[10$, p. 234].

EXAMPLE 4.1. We consider the following problem:

$$
\operatorname{minimize}(1 / 2) \int_{0}^{1} u^{2}(t) d t
$$

subject to

$$
\begin{aligned}
& \dot{x}(t)=u(t), \quad \text { given } x(0)>0, \\
& x(t) \geqq r(t) \quad \text { for all } t \in[0,1], \\
& u(t) \geqq 0 \quad \text { a.e. } t \in[0,1],
\end{aligned}
$$

where $x \in W_{1,1}^{1}([0,1]), u \in L_{\infty}^{1}([0,1])$ and $r$ is a given continuous function on $[0,1]$. 
We deal with this problem for the specific case; compare also [10, p. 234]:

$$
\begin{aligned}
& x(0)=1 / 2, \\
& r(t)= \begin{cases}-2 t^{2}+3 t & \text { if } 0 \leqq t \leqq 1 / 2, \\
1 & \text { if } 1 / 2<t \leqq 1\end{cases}
\end{aligned}
$$

We apply Corollary 4.1 to this problem. Put

$$
\Phi(t, x, u)=(1 / 2) u^{2}, \quad \phi(t, x, u)=u, \quad S(t, x)=r(t)-x .
$$

The Hamiltonian for this problem is

$$
H(t, x, u, \lambda(t))=(1 / 2) u^{2}-\lambda(t) u .
$$

By the local minimum principle, we obtain

$$
\begin{gathered}
\lambda(t)=\int_{t}^{1} d \psi(t)=\psi(1)-\psi(t), \\
\left(u_{0}(t)-\lambda(t)\right)\left(u-u_{0}(t)\right) \geqq 0 \quad \text { for all } u \geqq 0, \text { a.e. } t .
\end{gathered}
$$

Hence we have that

$$
\begin{aligned}
u_{0}(t)-\psi(1)+\psi(t) \geqq 0 & \text { a.e. } t, \\
\left(u_{0}(t)-\psi(1)+\psi(t)\right) u_{0}(t) & =0 \quad \text { a.e. } t .
\end{aligned}
$$

The complementary condition (4.16) becomes as follows:

$$
\Psi\left(\left\{t \mid r(t)<x_{0}(t)\right\}\right)=0
$$

This means that $\psi(t)$ varies only for those $t$ with $r(t)=x_{0}(t)$. It is easily seen that the following $\left(x_{0}, u_{0}, \psi\right)$ satisfies (4.38)-(4.40):

$$
\begin{aligned}
& x_{0}(t)= \begin{cases}t+1 / 2 & \text { if } 0 \leqq t \leqq 1 / 2, \\
1 & \text { if } 1 / 2 \leqq t \leqq 1,\end{cases} \\
& u_{0}(t)= \begin{cases}1 & \text { if } 0 \leqq t<1 / 2, \\
\text { arbitrary } & \text { if } t=1 / 2, \\
0 & \text { if } 1 / 2<t \leqq 1\end{cases} \\
& \psi(t)= \begin{cases}0 & \text { if } 0 \leqq t \leqq 1 / 2 \\
1 & \text { if } 1 / 2<t<1\end{cases}
\end{aligned}
$$

Define $\left(x_{1}, u_{1}\right)$ by

$$
\begin{aligned}
& x_{1}(t)= \begin{cases}4 t(t-1 / 2) & \text { if } 0 \leqq t \leqq 1 / 2, \\
0 & \text { if } 1 / 2 \leqq t \leqq 1,\end{cases} \\
& u_{1}(t)= \begin{cases}8 t-2 & \text { if } 0 \leqq t<1 / 2, \\
\text { arbitrary } & \text { if } t=1 / 2, \\
0 & \text { if } 1 / 2<t \leqq 1\end{cases}
\end{aligned}
$$

Then $\left(x_{1}, u_{1}\right)$ satisfies the conditions (4.12)-(4.15). Indeed, the conditions (4.12) and 
(4.14) are easily verified. Since the parameter set $I\left(z_{0}\right)$ of active constraints is equal to the closed interval $[1 / 2,1]$, we get that

$$
\max _{t \in[1 / 2,1]} S_{x}[t] x_{1}(t)=\max _{t \in[1 / 2,1]}\left(-x_{1}(t)\right)=0 .
$$

So the condition (4.13) is satisfied. Setting

$$
\hat{u}(t)= \begin{cases}4 t & \text { if } 0 \leqq t<1 / 2, \\ \text { arbitrary } & \text { if } t=1 / 2, \\ 0 & \text { if } 1 / 2<t \leqq 1\end{cases}
$$

we get that $u_{1}(t)=2\left(\hat{u}(t)-u_{0}(t)\right)$ and $\hat{u}(t) \geqq 0$ for all $t \in[0,1]$, which implies the condition (4.15). We now calculate the functional $E\left(t ; z_{0}, z_{1}\right)$. A necessary and sufficient condition for $t \in[0,1]$ and $\left\{t_{n}\right\} \subset[0,1]$ to satisfy (3.5) is that

$$
r\left(t_{n}\right)<x_{0}\left(t_{n}\right), \quad t_{n} \rightarrow t \quad \text { and } \quad x_{1}\left(t_{n}\right) /\left(r\left(t_{n}\right)-x_{0}\left(t_{n}\right)\right) \rightarrow+\infty
$$

as $n \rightarrow+\infty$. Since

$$
r\left(t_{n}\right)-x_{0}\left(t_{n}\right)=-2\left(t_{n}-1 / 2\right)^{2}
$$

and

$$
\begin{aligned}
x_{1}\left(t_{n}\right) /\left(r\left(t_{n}\right)-x_{0}\left(t_{n}\right)\right) & =4 t_{n}\left(t_{n}-1 / 2\right) /\left(-2\left(t_{n}-1 / 2\right)^{2}\right) \\
& =-2 t_{n} /\left(t_{n}-1 / 2\right) \rightarrow+\infty \quad \text { as } n \rightarrow+\infty,
\end{aligned}
$$

the condition (4.41) is equivalent to that $t=1 / 2$ and $t_{n} \uparrow 1 / 2$. Hence $T_{0}\left(z_{0}, z_{1}\right)=\{1 / 2\}$. Moreover, for these $\left\{t_{n}\right\}$, it holds that

$$
\underset{n \rightarrow+\infty}{\limsup } x_{1}^{2}\left(t_{n}\right) / 4\left(x_{0}\left(t_{n}\right)-r\left(t_{n}\right)\right)=\lim _{n \rightarrow+\infty} 16 t_{n}^{2}\left(t_{n}-1 / 2\right)^{2} / 8\left(t_{n}-1 / 2\right)^{2}=\lim _{n \rightarrow+\infty} 2 t_{n}^{2}=1 / 2
$$

Consequently, we have

$$
E\left(t ; z_{0}, z_{1}\right)= \begin{cases}-\infty & \text { if } 0 \leqq t<1 / 2 \\ 1 / 2 & \text { if } t=1 / 2 \\ 0 & \text { if } 1 / 2<t \leqq 1\end{cases}
$$

Let $\Psi^{*}$ be the completion of the measure $\Psi$ induced from $\psi$. Then we obtain that

$$
\int_{0}^{1} E\left(t ; z_{0}, z_{1}\right) d \Psi^{*}(t)=1 / 2>0 .
$$

Moreover,

$$
\begin{gathered}
\int_{0}^{1}\left(x_{1}(t), u_{1}(t)\right)^{*}\left(\begin{array}{l}
\Phi_{x x}[t], \Phi_{x u}[t] \\
\Phi_{u x}[t], \Phi_{u u}[t]
\end{array}\right)\left(\begin{array}{l}
x_{1}(t) \\
u_{1}(t)
\end{array}\right) d t \\
=\int_{0}^{1}\left(x_{1}(t), u_{1}(t)\right)^{*}\left(\begin{array}{ll}
0 & 0 \\
0 & 1
\end{array}\right)\left(\begin{array}{l}
x_{1}(t) \\
u_{1}(t)
\end{array}\right) d t \\
=\int_{0}^{1} u_{1}^{2}(t) d t=\int_{0}^{1 / 2}(8 t-2)^{2} d t=2 / 3
\end{gathered}
$$


and

$$
\int_{0}^{1} x_{1}(t) * S_{x x}[t] x_{1}(t) d \psi(t)=\int_{0}^{1} 0 d \psi(t)=0
$$

Hence we have

$$
\begin{aligned}
& \int_{0}^{T}\left(x_{1}(t), u_{1}(t)\right)^{*}\left(\begin{array}{l}
H_{x x}[t], H_{x u}[t] \\
H_{u x}[t], H_{u u}[t]
\end{array}\right)\left(\begin{array}{l}
x_{1}(t) \\
u_{1}(t)
\end{array}\right) d t \\
& \quad+\int_{0}^{T} x_{1}(t)^{*} S_{x x}[t] x_{1}(t) d \psi(t)+2 \int_{0}^{T} E\left(t ; z_{0}, z_{1}\right) d \Psi^{*}(t) \\
& =2 / 3+0+1=5 / 3 \geqq 0,
\end{aligned}
$$

which shows the second-order necessary condition (4.19) is satisfied at $\left(x_{0}, u_{0}\right)$ for the particular direction $\left(x_{1}, u_{1}\right)$.

\section{Conclusion}

In Theorem 4.1, we have derived a new second-order necessary condition, as well as the usual local minimum principle, for the optimal control problem with state constraints. It should be noted that the necessary condition involves the extra term $2 \int_{0}^{T} E\left(t ; z_{0}, z_{1}\right) d \Psi^{*}(t)$ besides the second derivative of the Lagrange function. The extra term is regarded as the result of the envelope-like effect of the infinitely many state constraints in the control problem. As noted in Remark 4.1, the extra term disappear when we are concerned with the finitely many state constraints.

\section{Acknowledgement}

The author would like to thank Professor N. Furukawa for his helpful advice.

\section{References}

[1] Dem'yanov, V. F.: Second-order directional derivatives of a function of the maximum, Cybernetics, 9 (1975), 797--800.

[2] Dubovitskil, A. Ya. and Milyutin, A. A.: Problems of the extremum with restrictions, Zh. Vychisl. Mat. i Mat. Fiz., 3 (1965).

[3] Furukawa, N. and Yoshinaga, Y.: Higher order variational sets, variational derivatives and higher order necessary conditions in abstract mathematical programming, Bulletin of Informatics and Cybernetics, 23 (1988), 9-40.

[4] Gilbert, E. G. and Bernstein, D. S.: Second-order necessary conditions in optimal control: Accessoryproblem results without normality conditions, Journal of Optimization Theory and Applications, 41 (1983), 75-106.

[5] GiRSANOV, I. V.: Lectures on mathematical theory of extremum problems, Lecture notes in economic and mathematical systems, (Springer, Berlin), 67 (1972). 
[6] HofFmAN, K. H. and KORNSTAEDT, H. J.: Higher order necessary conditions in abstract mathematical programming, Journal of Optimization Theory and Applications, 26 (1978), 533-569.

[7] JACOBSON, D. H., Lele, M. M. and SPEYER, J. L.: New necessary conditions of optimality for control problems with state-variable inequality constraints, Journal of Mathematical Analysis and Applications, 35 (1971), 255-284.

[8] KaWASAKI, H.: An envelope-like effect of infinitely many inequality constraints on second-order necessary conditions for minimization problems, Mathematical Programming, 41 (1988), 73-96.

[9] KAWASAKI, H.: Second order necessary optimality conditions for minimizing a sup-type function, to appear in Mathematical Programming.

[10] Luenberger, D. G.: Optimization by vector space methods, John Wiley \& Sons, Inc., (1969).

[11] MaruYama, Y.: Second-order necessary conditions for nonlinear optimization problems in Banach spaces and their application to an optimal control problem, to appear in Mathematics of Operations Research.

[12] MaruYama, Y.: Second-order necessary conditions for nonlinear optimization problems in Banach spaces by the use of Neustadt derivative, Submitted.

[13] MAURER, H.: First and second order sufficient optimality conditions in mathematical programming and optimal control, Mathematical Programming Study, 14 (1981), 163-177.

[14] NoRRIS, D. O.: Nonlinear programming applied to state-constrained optimization problems, Journal of Mathematical Analysis and Applications, 43 (1973), 261-272.

[15] WARGA, J.: A second-order Lagrangian condition for restricted control problems, Journal of Optimization Theory and Applications, 24 (1978), 475-483.

Received February 1, 1989

Communicated by N. Furukawa 